ISSN: 2640-1193

Open Access | Research Article

\title{
False Positive Rifampicin Resistance among HIV Patients attending Bahati Comprehensive Care Centre, Nairobi, Kenya
}

\author{
Lucy Obonyo Nyang'au ${ }^{1 *}$; Evans Amukoye ${ }^{2}$; Stanley Kangethe ${ }^{1}$; Jackson Onyuka ${ }^{1}$ \\ ${ }^{1}$ Mount Kenya University, Thika, Nairobi, Kenya \\ ${ }^{2}$ Kenya Medical Research Institute, Nairobi, Kenya
}

\section{*Corresponding Author(s): Lucy Obonyo Nyang'au}

Department of Medical Laboratory Sciences, Mount Kenya University, P.O Box 342-01000, Thika, Kenya

Tel: +254-072 281 6265;

Email: lucynyangau@yahoo.com

Received: May 23, 2020

Accepted: Jul 10, 2020

Published Online: Jul 16, 2020

Journal: Journal of Tuberculosis

Publisher: MedDocs Publishers LLC

Online edition: http://meddocsonline.org/

Copyright: @ Nyang'au LO (2020). This Article is distributed under the terms of Creative Commons Attribution 4.0 International License

Keywords: GeneXpert MTB/RIF assay; Mycobacterium tuberculosis; Rifampicin resistant false positives

\begin{abstract}
Introduction: Tuberculosis is a chronic airborne and infectious disease which remains a leading cause of death in people living with Human Immunodeficiency Virus (HIV). Globally in 2018, there were 251,000 documented cases of death in HIV positive people. Timely diagnosis of tuberculosis and detection of drug resistance to TB drugs in such patients remains a challenge because of non specific presentation and disseminated nature of the disease. GeneXpert MTB/RIF assay is a molecular technique capable of simultaneously diagnosing tuberculosis and providing susceptibility status of rifampicin which is a surrogate marker of multidrug resistant tuberculosis. Despite the improved detection capacity in both tuberculosis and rifampicin susceptibility status previous studies have reported on false positive rifampicin resistance associated with Xpert MTB/RIF assay.
\end{abstract}

Objective: This study sought to determine the prevalence of false positive rifampicin resistance among HIV patients attending Bahati comprehensive care centre.

Materials and Methods: In a seven month period a retrospective cohort study was conducted among consented 346 people living with human immunodeficiency virus (HIV) attending a comprehensive care centre in Nairobi, Kenya. Detection and isolation of Mycobacterium tuberculosis from patient sputa was done using Xpert MTB/RIF assay and BACTEC MGIT 960 machine, on the other hand first line drug susceptibility testing was done by use of BACTEC MGIT 960 machine. All collected data was analyzed using Statistical Package for the Social Sciences (SPSS) version 20.0.

Results: Of the 72(93.5\%) Xpert MTB/RIF assay culture positive isolates subjected to drug susceptibility, results revealed $4(6.5 \%)$ and $1(10 \%)$ false rifampicin resistant cases in the non isoniazid preventive therapy and isoniazid preventive therapy (non-IPT and IPT) arms respectively; $\mathrm{OR}=1.583[95 \% \mathrm{C} . \mathrm{I}=0.159-15.813] ; \mathrm{P}=0.538$. There were no significant differences regarding false rifampicin resistant

Cite this article: Nyang'au LO, Amukoye E, Kangethe S, Onyuka J. False Positive Rifampicin Resistance among HIV Patients attending Bahati Comprehensive Care Centre, Nairobi, Kenya. J Tuberc. 2020; 3(1): 1018. 
cases in relation to patient treatment status, age and gender. Significant differences regarding false rifampicin resistant cases were recorded in relation to the Mycobacterium tuberculosis (MTB) load levels; MTB detected low 2 (50\%) and MTB detected very low 2 (100\%), revealed significance to false rifampicin resistant cases $(P=0.001)$.

Conclusion: Samples with very low and low MTB detection levels were more prone to false positive rifampicin resistance. Such results should be interpreted with caution and confirmed with phenotypic drug susceptibility testing. There was no significant association between false positive rifampicin resistance and the patient treatment status, age or gender of the patients

\section{Introduction}

Mycobacterium tuberculosis the causative agent for tuberculosis (TB) is a core cause of increased morbidity and mortality especially among people living with HIV and a global public health concern [1]. The control of tuberculosis is aggravated by the emergence of drug resistance strains and the human immune deficiency virus [2]. Early and rapid diagnosis of TB and appropriate use of recommended therapy is essential in control of the emergence and spread of multi-drug resistant tuberculosis (MDR-TB) and extremely drug resistant tuberculosis (XDR-TB) strains [2]. Use of conventional techniques for diagnosis of TB and drug resistance may lead to delayed treatment, worse clinical outcomes and increased transmission because these methods require long durations for result outcome [3]. Conventionally diagnosis of MDR-TB requires mycobacterial culture and phenotypic drug susceptibility testing, these techniques require complex laboratories, they are labor intensive, and takes at least 1-3 months before results are available [4]. In 2010, WHO endorsed Xpert MTB/RIF assay as the initial test for simultaneous diagnosis of Mycobacterium tuberculosis and rifampicin resistance, rifampicin is one of the principal firstline anti-TB drugs and a potent marker for MDR-TB which plays an important role in the treatment of rifampicin sensitive tuberculosis [5]. GeneXpert assay is an automated cartridge based assay designed to simultaneously detect Mycobacterium tuberculosis and rifampicin resistance directly on clinical specimens using heminested real time polymerase chain reaction (PCR) which target the $81 \mathrm{bp}$ rifampicin resistance determining region (RRDR) of the rpoB gene $[6,7]$. The assay platform automatically gives results within 2 hours of testing, use of Xpert MTB/RIF assay leads to rapid diagnosis of both Mycobacterium tuberculosis and rifampicin resistance, this could reduce the morbidity, mortality and transmission of both drug susceptible and drug resistant TB [6]. Rifampicin being a surrogate marker of MDR-TB its early detection is essential for early management of cases and prevention of resistant strains transmission [8]. Also it has important implications for both the individual's health as well as the community. MDR-TB defined as resistance to at least isoniazid and rifampicin is associated with worse clinical outcomes, complications and increased transmission [8]. Global control of tuberculosis has been faced with challenges which include among others drug resistance and HIV; this has prompted an urgent need for timely and effective diagnosis method of both tuberculosis and drug susceptibility testing [8].

Materials and methods

\section{Study design}

A retrospective cohort study design was employed whereby eligible HIV positive participants (with or without use of isoniazid preventive therapy) were recruited through cluster random sampling, those who consented were included in the study.

\section{Inclusion and exclusion criteria}

Patients who had signs and symptoms of tuberculosis, above 15 years of age, at one year post isoniazid preventive therapy (IPT), were on HIV care, and accepted consent were included in the study. While patients with unclear IPT and age records, refusal to consent, unable to produce sputum were excluded from the study.

\section{Sputum Collection}

Sputa collected according to standard operating procedures and in well labeled $50 \mathrm{ml}$ sterile conical tubes were processed according to the manufacturer guidelines for GeneXpert MTB/ RIF assay and cultured using BACTEC MGIT 960 machine.

\section{Laboratory Procedures}

\section{Identification of Mycobacterium tuberculosis}

Xpert MTB/RIF assay reagent (buffer) was added into quality sputum samples in $50 \mathrm{ml}$ sterile falcon tubes in a ratio 1:2 for liquefaction and lysis of the mycobacteria [9]. The mixture was gently but vigorously mixed using a vortexer and allowed to sit for $15 \mathrm{~min}$ before being mixed again and allowed to sit for another $5 \min [9]$.

Using sterile pasture pipette $2 \mathrm{ml}$ of the processed sample was loaded into the Xpert MTB/RIF assay cartridge and test started on Xpert MTB/RIF assay machine platform. The assay is an automatic process with internal quality controls; the sample processing control which serves to verify that lysis of Mycobacterium tuberculosis has taken place, sample preparation is adequate and helps to detect any inhibitor of polymerase chain reaction [4,9]. Sample processing control must be positive when the result reads Mycobacterium tuberculosis not detected, while it can be negative or positive when the result is Mycobacterium tuberculosis detected. The probe check serves to measure fluorescence signal, rehydrating the beads and checking stability of the probe and dye [9]. Once the tests were complete, results were either of the following; Mycobacterium tuberculosis not detected, Mycobacterium tuberculosis detected very Low, Low, Medium or High. In this case the rifampicin resistance can be either detected or not detected. The test results can also be in form of an error or invalid status, in this case the test must be repeated [9].

\section{Culture of processed samples}

Sputa collected in sterile $50 \mathrm{ml}$ conical tubes were decontaminated according to the standard operating procedures using equal volumes of sputa and sodium hydroxide- N-acetyl-LCystein(NAOH-NALC) method [10,11]. Upon decontamination of the sputa the pellet obtained were re-suspended in $2 \mathrm{ml}$ of buffered phosphate saline ( $\mathrm{PH} \mathrm{6.8),} \mathrm{which} \mathrm{neutralizes} \mathrm{the} \mathrm{sodium}$ hydroxide and dilutes the homogenate to lessen the viscosity and specific gravity prior to centrifugation $[10,11]$. The pellets were used to prepare smears for staining by Ziehl-Nielsen staining and inoculating the liquid media Mycobacterium Growth Indicator Tube (MGIT) 960 tubes. The inoculated tubes were incubated along with negative control (un-inoculated MGIT media) and positive control (H37Rv ATCC 27294) [10]. All the inoculated MGIT tubes were scanned and the caps tightly closed before being entered into the BACTEC MGIT 960 machine. The incuba- 
tion was done at $37^{\circ} \mathrm{C}$ until the instrument flagged them positive and after a maximum of six weeks, the instrument flagged the tubes negative only if there was no growth at $37^{\circ} \mathrm{C}[10]$. The instrument signaled a positive tube by indicating green light at the exact location in the drawer of the instrument. The isolates from MGIT 960 were subjected to confirmative identification of MTB using BD MGIT TBC, whereby confirmed positive test for MTB was indicative of active disease.

\section{Quality control}

Un-inoculated Mycobacteria growth indicator tube (MGIT) (negative control), and H37Rv ATCC, 27294 (positive control) were processed and included during the test run. Purity of bacterial suspensions used was checked by culture on blood agar [12].

Quality control of acid fast bacilli (AFB) smears was done by including a positive and negative control slide with each batch of slides stained and with every fresh batch of stain. The smears were prepared from positive cultures of MTB H37RvATCC 27294 used as positive control while Escherichia Coli bacterial suspension was used as negative control [12]. The controls were examined before the clinical specimens.
First line drug susceptibility testing using MGIT technique: Drug susceptibility testing for first line TB drugs was done for the MTB strains, using BACTEC MGIT 960 machine. This was done in accordance with the standard operating procedures provided by the manufacturer. Final concentrations were $1.0 \mu \mathrm{g} / \mathrm{ml}$ for streptomycin (S), $0.1 \mu \mathrm{g} / \mathrm{ml}$ for isoniazid (INH), $1.0 \mu \mathrm{g} / \mathrm{ml}$ for rifampicin (R), $5.0 \mu \mathrm{g} / \mathrm{ml}$ for ethambutol (E) and $100 \mu \mathrm{g} / \mathrm{ml}$ for pyrazinamide (PZA) [12]. The results were automatically interpreted by the BACTEC MGIT 960 instrument and reported as either susceptible, resistant or error.

\section{Statistical analysis}

Demographic and laboratory data was entered and analyzed by Statistical Package for the Social Sciences (SPSS) version 20.0 statistical software. Pearson's Chi-square test and Fisher's exact test were applied to determine the differences in proportion for both groups in IPT status, type of patients, demographics and MTB detection levels in geneXpert against the rifampicin false positives. The results were presented by appropriate tabulations based on the determined variables, (OR) odds ratio with 95\% confidence interval $(\mathrm{Cl})$ and the corresponding $\mathrm{p}$ values. The threshold for statistical significance was set at $\mathrm{P} \leq 0.05$.

Table 1: Prevalence of Rifampicin Resistant False Positives among study patients.

\begin{tabular}{|c|c|c|c|c|c|c|}
\hline \multirow[t]{2}{*}{ Variables } & \multirow{2}{*}{$\begin{array}{l}\text { Total } \\
\text { (N) }\end{array}$} & \multicolumn{2}{|c|}{ True Xpert results n (\%) } & \multirow{2}{*}{$\begin{array}{c}\text { False positives n (\%) } \\
\text { Xpert-RR/DST-RS }\end{array}$} & \multirow[t]{2}{*}{ OR $(95 \% \mathrm{Cl})$} & \multirow[t]{2}{*}{ P Value } \\
\hline & & XpertRR-/DST-RR & Xpert-RS/DST-RS & & & \\
\hline \multicolumn{7}{|l|}{ IPT Status } \\
\hline Non-IPT Patients & 62 & $3(4.8)$ & $55(88.7)$ & $4(6.5)$ & \multirow{2}{*}{$1.583(0.159-15.813)$} & \multirow{2}{*}{0.538} \\
\hline IPT Patients & 10 & $0(0)$ & $9(90)$ & $1(10)$ & & \\
\hline \multicolumn{7}{|l|}{ Type of patients } \\
\hline New Patients & 57 & $2(3.5)$ & $50(87.7)$ & $5(8.8)$ & \multirow{2}{*}{ UD } & \multirow{2}{*}{0.293} \\
\hline RT Patients & 15 & $1(6.7)$ & $14(93.3)$ & $0(0)$ & & \\
\hline \multicolumn{7}{|l|}{ MTB Detection levels } \\
\hline MTB Detected High & 25 & $0(0)$ & $25(100)$ & $0(0)$ & & \\
\hline MTB Detected Medium & 41 & $3(7.3)$ & $37(90.2)$ & $1(2.4)$ & UD & 0.999 \\
\hline MTB Detected Low & 4 & $0(0)$ & $2(50)$ & $2(50)$ & UD & 0.001 \\
\hline MTB Detected Very Low & 2 & $0(0)$ & $0(0)$ & $2(100)$ & UD & 0.001 \\
\hline \multicolumn{7}{|l|}{ Age(Years) } \\
\hline$<20$ & 4 & $1(25)$ & $3(75)$ & $0(0)$ & & \\
\hline $20-39$ & 42 & $1(24)$ & 39 (92.9) & $2(4.8)$ & $0.722(0.422-1.271)$ & 0.617 \\
\hline $40-59$ & 23 & $0(0)$ & $20(87.7)$ & $3(13)$ & UD & 0.999 \\
\hline $60+$ & 3 & $1(33.3)$ & $2(66.7)$ & $0(0)$ & UD & 0.999 \\
\hline \multicolumn{7}{|l|}{ Gender } \\
\hline Female & 28 & $2(7.1)$ & $24(85.7)$ & $2(7.1)$ & \multirow{2}{*}{$0.915(0.143-5.858)$} & \multirow{2}{*}{0.781} \\
\hline Male & 44 & $1(2.3)$ & 40 (90.9) & $3(4.2)$ & & \\
\hline
\end{tabular}

RR: Rifampicin resistance; RS: Rifampicin sensitive; DST: Drug susceptibility testing; OD: Odds ratio; C.I: Confidence interval 
Table 2: Distribution of Rifampicin resistant false positives among Study Patients based on IPT status, age and gender.

\begin{tabular}{|c|c|c|c|c|c|c|c|}
\hline \multirow[b]{2}{*}{ IPT Status } & \multirow[b]{2}{*}{ Gender } & & & \multicolumn{2}{|c|}{ True rifampicin results } & \multirow{2}{*}{$\begin{array}{c}\text { False rifampicin } \\
\text { resistance } \\
\begin{array}{c}\text { Xpert-RR / } \\
\text { DST-RS }\end{array}\end{array}$} & \multirow{2}{*}{ Total } \\
\hline & & & & $\begin{array}{c}\text { Xpert-RR / } \\
\text { DST-RR }\end{array}$ & $\begin{array}{c}\text { Xpert-RS/ } \\
\text { DST-RS }\end{array}$ & & \\
\hline \multirow{14}{*}{ IPT Patients } & \multirow{4}{*}{ Male } & \multirow{3}{*}{ Age (Years) } & $60+$ & $0(0 \%)$ & $1(10 \%)$ & $0(0 \%)$ & 1 \\
\hline & & & $40-59$ & $0(0 \%)$ & $2(20 \%)$ & $0(0 \%)$ & 2 \\
\hline & & & $20-39$ & $0(0 \%)$ & $2(20 \%)$ & $1(10 \%)$ & 3 \\
\hline & & Total & & $0(0 \%)$ & $5(50 \%)$ & $1(10 \%)$ & 6 \\
\hline & \multirow{5}{*}{ Female } & \multirow{4}{*}{ Age (Years) } & $60+$ & $0(0 \%)$ & $1(10 \%)$ & $0(0 \%)$ & 1 \\
\hline & & & $40-59$ & $0(0 \%)$ & $1(10 \%)$ & $0(0 \%)$ & 1 \\
\hline & & & $20-39$ & $0(0 \%)$ & $1(10 \%)$ & $0(0 \%)$ & 1 \\
\hline & & & $<20$ & $0(0 \%)$ & $1(10 \%)$ & $0(0 \%)$ & 1 \\
\hline & & Total & & $0(0 \%)$ & $4(40 \%)$ & $0(0 \%)$ & 4 \\
\hline & \multirow{5}{*}{ Total } & \multirow{4}{*}{ Age (Years) } & $60+$ & $0(0 \%)$ & $2(20 \%)$ & $0(0 \%)$ & 2 \\
\hline & & & $40-59$ & $0(0 \%)$ & $3(30 \%)$ & $0(0 \%)$ & 3 \\
\hline & & & $20-39$ & $0(0 \%)$ & $3(30 \%)$ & $1(10 \%)$ & 4 \\
\hline & & & $<20$ & $0(0 \%)$ & $1(10 \%)$ & $0(0 \%)$ & 1 \\
\hline & & Total & & $0(0 \%)$ & $9(90 \%)$ & $1(10 \%)$ & 10 \\
\hline \multirow{14}{*}{ Non IPT patient } & \multirow{5}{*}{ Male } & \multirow{4}{*}{ Age (Years) } & $60+$ & $1(1.6 \%)$ & $0(0 \%)$ & $0(0 \%)$ & 1 \\
\hline & & & $40-59$ & $0(0 \%)$ & $11(17.7 \%)$ & $1(1.6 \%)$ & 12 \\
\hline & & & $20-39$ & $0(0 \%)$ & $23(37.1 \%)$ & $1(1.6 \%)$ & 24 \\
\hline & & & $<20$ & $0(0 \%)$ & $1(1.6 \%)$ & $0(0 \%)$ & 1 \\
\hline & & Total & & $1(1.6 \%)$ & $35(56.5 \%)$ & $2(3.2 \%)$ & 28 \\
\hline & \multirow{4}{*}{ Female } & \multirow{3}{*}{ Age (Years) } & $40-59$ & $0(0 \%)$ & $6(9.7 \%)$ & $2(3.2 \%)$ & 8 \\
\hline & & & $20-39$ & $1(1.6 \%)$ & $13(21 \%)$ & $0(0 \%)$ & 14 \\
\hline & & & $<20$ & $1(1.6 \%)$ & $1(1.6 \%)$ & $0(0 \%)$ & 2 \\
\hline & & Total & & $2(3.2 \%)$ & $20(32.3 \%)$ & $2(3.2 \%)$ & 24 \\
\hline & \multirow{5}{*}{ Total } & \multirow{4}{*}{ Age (Years) } & $60+$ & $1(1.6 \%)$ & $0(0 \%)$ & $0(0 \%)$ & 1 \\
\hline & & & $40-59$ & $0(0 \%)$ & $17(27.4 \%)$ & $3(4.8 \%)$ & 20 \\
\hline & & & $20-39$ & $1(1.6 \%)$ & $36(58.1 \%)$ & $1(1.6 \%)$ & 38 \\
\hline & & & $<20$ & $1(1.6 \%)$ & $2(3.2 \%)$ & $0(0 \%)$ & 3 \\
\hline & & Total & & $3(4.8 \%)$ & $55(88.7 \%)$ & $4(6.5 \%)$ & 62 \\
\hline Total & & & & 3 & 64 & 5 & 72 \\
\hline
\end{tabular}

IPT: Isoniazid preventive therapy; DST: Drug susceptibility testing; RR: Rifampicin resistant; RS: Rifampicin sensitive 


\section{Results}

\section{Prevalence of Rifampicin Resistant False Positives}

Of the 72 isolates subjected to drug susceptibility testing, 4(6.5\%) and 1(10\%); OR=1.583 [95\% C.I= 0.159-15.813]; $\mathrm{P}=0.538$ false positive rifampicin resistance cases were identified in the non-IPT and IPT arms respectively; with the new and retreatment patients having $5(8.8 \%)$ and $0(0 \%)(P=0.293)$; cases respectively as indicated in (Table 1 ). This study revealed insignificant differences in false positive rifampicin resistance cases regarding the IPT status of the patients and the patient treatment status. 5(6.9\%) of the rifampicin resistant cases with Xpert MTB/RIF assay were susceptible on phenotypic drug susceptibility testing using BACTEC MGIT 960 as the reference standard (Table 1). The study revealed significant difference among Mycobacterium tuberculosis (MTB) detection levels of low and very low respectively $(\mathrm{P}=0.001)$ (Table 1$)$. The findings indicated that there was no significant difference in gender with regard to false rifampicin resistance; $O R=0.915$ [95\% C.I =0.143-5.858]; $\mathrm{P}=0.781$ (Table 1). Further the findings indicated there were $1(10 \%)$ and $0(0 \%), 2(3.2 \%)$ and $2(3.2 \%)$ false positive rifampicin resistant cases among males and females in the IPT and Non-IPT arms respectively (Table 2). In the age category (20-39) $1(10 \%)$ and $0(0)$ males and females had false positive rifampicin resistance in the IPT arm while $1(1.6 \%)$ and $0(0 \%)$ males and females had false positive rifampicin resistance in the Non-IPT arm (Table 2). In the age category (40-59) $0(0 \%)$ and $0(0 \%)$ male and female cases from the IPT arm had false positive rifampicin resistance while $1(1.6 \%)$ and $2(3.2 \%)$ male and female cases from the Non-IPT arm had false positive rifampicin resistance (Table 2). Overall the study findings revealed significant association between bacterial load level and false rifampicin resistance cases, where low and very low bacterial loads were associated with false rifampicin resistant cases $(P=0.001)$. There was no significant association between false rifampicin resistant cases and IPT status, patient treatment status, gender or age of the patient.

\section{Discussion}

One of the most critical steps in TB management is the prompt and accurate laboratory diagnosis of tuberculosis and drug resistance susceptibility testing. This need has led to molecular diagnostic methods becoming commonly used and taking a complementary role along with conventional techniques [5]. Accurate and prompt diagnosis of both tuberculosis and drug resistance to either of the first line TB drugs is important in selection of appropriate regimen to which the strain is susceptible and in timely initiation of treatment. At the same time early diagnosis of tuberculosis and drug resistance to any of the first line TB drugs facilitates appropriate measures to prevent transmission [13]. For the patient a false rifampicin resistance result may result in overtreatment with more toxic and less effective second line TB drugs and also unnecessary prolonged treatment [14].

The current study findings revealed $6.9 \%$ false positive rifampicin resistance cases, among low and very low mycobacterial levels, this is supported by previous studies in other settings. The current study findings were markedly low compared with those recorded in previous studies conducted in Haiti (62.8\%) [8], India (66\%) [15], Korea (20\%) [16], Australia (31\%) [17], but they were low compared to previous findings in Kenya (12\%) [18] and Turkey (11.7\%) [19]. On the other hand the findings were higher than those documented previously in Egypt (2.0\%)
[20], Pakistan (0.8\%) [21], China (0.41\%) [22], South Africa (0.9\%) [13], but comparable with previous findings in studies conducted in Vietnam (6\%) [23] and India (6\%) [24]. The current findings confirm with other previous findings that rifampicin resistant false positives are significantly associated with low and very low mycobacterial load. In such cases microbiologists and clinicians must be aware of the limitations of the assay when interpreting the Xpert MTB/RIF test results, for proper managements of the patients.

\section{Conclusions}

False positivity to rifampicin resistance was significantly associated with low and very low mycobacterial levels. This implies that growth based drug susceptibility testing remains an integral diagnostic test to confirm molecular results, to avoid unwarranted second-line TB treatment which is costly, leads to exposure to toxic drugs, stigma, loss of jobs and family separation. The findings of this study have important policy implications; need for revision and standardization of TB management guidelines to avoid exposing patients to unwarranted second line TB treatment, which is very costly, less effective and as high toxicity. On the other hand the technical and clinical capacities should be continually build to keep pace with the ever changing technology.

\section{Recommendation}

Those patients with very low and low Mycobacterium tuberculosis detection levels with rifampicin resistance detected should be commenced on second-line treatment based on phenotypic drug susceptibility results. On the other hand there is need for further studies to determine specificity for the detection of rifampicin resistance, depending on the bacterial load in clinical samples for evaluation of the future Xpert MTB/RIF versions.

\section{Acknowledgement}

The authors sincerely thank Central Reference Laboratory, Bahati Comprehensive Care Centre staffs and all those who directly or indirectly contributed to the success of this study.

\section{References}

1. Boehme CC, Nabeta P, Hillemann D, Nicol MP, Shenai S, Krapp F. Rapid molecular detection of tuberculosis and rifampicin resistance. New England Journal of Medicine. 2010; 363(11): 10051015.

2. Mathys V, VandeVyvere M, DeDroogh E, Soetaert K, Groenen G. False positive rifampicin resistance on Xpert MTB/RIF caused by a silent mutation in the rpoB gene. International Journal of Tuberculosis and Lung Disease. 2014; 18: 1255-1257.

3. Helb D, Jones M, Story E, Boehme C, Wallace E, Ho K. Rapid detection of Mycobacterium tuberculosis and rifampicin resistance by use of on demand, near patient technology. Journal of Clinical Microbiology. 2010; 48(1): 229-37.

4. Van Rie A, Mellet K, John MA, Scott L, Page-Shipp L, Dansey H. False positive rifampicin resistance on Xpert MTB/RIF: A case report and clinical implications. International Journal of Tuberculosis and Lung Disease. 2012; 16(2): 206-8.

5. Boehme CC, Nicol MP, Nabeta P, Michael JS, Gotuzzo E, Tahirli R, Feasibility diagnostic accuracy and effectiveness of decentralized use of the Xpert MTB/RIF test for diagnosis of tuberculosis and multidrug resistance: $A$ multicentre implementation study. Lancet. 2011; 377: 1495-1505. 
6. Chang K, Lu W, Wang J, Zhang K, Jia S, Li F. Rapid and effective diagnosis of tuberculosis and rifampicin resistance with Xpert MTB/RIF assay: A meta-analysis. Journal of Infection. 2012; 64(6): 580-588.

7. Blakemore R, Story E, Helb D, Kop J, Banada P, Owens MR. Evaluation of the analytical performance of the Xpert MTB/RIF assay. Journal of Clinical Microbiology. 2010; 48(7): 2495-2501.

8. Ocheretina O, Byrt E, Mabou MM, Royal-Mardi G, Merveille YM, Rouzier V, Fitzgerald DW, Pape JW. False positive RifampicinResistant Results with Xpert MTB/RIF version 4 assay in clinical samples with a low bacterial load. Diagnostic Microbiology Infectious Disease. 2016.

9. Rakha EB, Abdel Razek Abdel Hakeem M. GeneXpert MTB/RIF assay: A revolutionizing method for rapid molecular detection of Mycobacterium tuberculosis in comparison to other conventional methods. International Journal of current Microbiology and Applied Sciences. 2017; 2319-7706, 6(10) Pp. 2573-2580.

10. Lee JJ, Lin CB, Wang JD. Comparative evaluation of the BACTEC MGIT 960 system with solid medium for isolation of mycobacteria. International Journal of Tuberculosis and Lung Disease, 2003; 7: 569-574.

11. Nyang'au LO, Ng'ang'a Z, Amukoye E. First line Anti- Tuberculosis drug resistance among Human Immunodeficiency Virus Infected Patients attending Maryland Comprehensive Care Centre, Mathare 4A, Nairobi Kenya. International Journal of Sciences: Basic and Applied Research. 2014; 661-668.

12. Yan JJ, Huang AH, Tsai SH. Comparison of the MB/BacT and BACTEC MGIT 960 system for recovery of Mycobacteria from clinical specimens. Diagnostic Microbiology and Infectious Diseases, 2000; 37: 25-30.

13. Theron G, Peter J, Van-Zyl-Smit R, Mishra G, Streicher E, Murray S. Evaluation of the Xpert MTB/RIF assay for the diagnosis of pulmonary tuberculosis in a high prevalence setting. American Journal of Respiratory and Critical Care Medicine. 2011; 184: 132-140.

14. Zignol M, Dean AS, Falzon D. Twenty years of global surveillance of anti-tuberculosis-drug resistance. New England Journal of Medicine. 2016; 375: 1081-1089.

15. Sanker P, Kottuthodi RP, Ambika AP, Santhosh VT, Balakrishnan $R$, Mrithunjavan SK. Predictable Repeatability issues with GeneXpert MTB/RIF (version 4). Derived Rifampicin Resistant Tuberculosis results from South India: Appreciating the limits of a technological marvel. Biomedical and Biotechnology Research Journal (BBRJ). 2017.

16. Kim CH, Hyun GI, Hwang YI, Kim DG, Lee CY, Lee MG. Identification of Mycobacterium tuberculosis and Rifampicin resistance in clinical specimens using the Xpert MTB/RIF Assay. Annals of
Clinical and Laboratory Science. 2015; 45(1): 32-38.

17. Williamson DA, Basu I, Bower J, Freeman JT, Henderson G, Roberts SA. An evaluation of the Xpert MTB/RIF assay and detection of false positive rifampicin resistance in Mycobacterium tuberculosis. Diagnostic Microbiology and Infectious Disease. 2012; 74(2): 207-209.

18. Muia PK, Ngugi MP Mburu DN. Performance of GeneXpert in detecting tuberculosis and rifampicin resistance in patients attending Kitui County Hospital Kenya. Journal of Tropical Diseases and Public Health. 2017; 5: 4.

19. Cayci YT, Bilgin K, Coban YA, Birinci A, Durupinari B. (2017). An evaluation of false positive rifampicin resistance on the Xpert MTB/RIF. Memorias do Instituto Oswaldo Cruz, Rio de janeiro. 2017; 112 (11):756-759.

20. Omar A, Yousef Ahmed AA E, Hosny M. Valuing the use of geneXpert test as an unconventional approach to diagnose pulmonary tuberculosis. Egyptian Journal of Bronchology. 2019; 13: 403-407.

21. Saeed M, Iram S, Hussain S, Ahmed A, Akbar M, Aslam M. GeneXpert: A new tool for detection of rifampicin resistance in $M y$ cobacterium tuberculosis, Journal of Pakistan Medical Association. 2017; 67(2): 270-274.

22. Tang T, Liu F, Lu X, Huang Q. Evaluation of GeneXpert MTB/RIF for detecting Mycobacterium tuberculosis in a hospital in China. Journal of International Medical Research. 2017; 45(2): 816822.

23. Nguyen P, Hung NV. Report on false positive rifampicin resistance on GeneXpert MTB/RIF in Vietnam. 2013

24. Ajbani K, Naik S, Kazi M, Shetty A, Rodrigues C. Interpreting very low Mycobacterium tuberculosis detected on Xpert Mycobacterium tuberculosis/rifampicin. Lung India. 2019; 36(6): 555-557. 